\title{
Dislocations and melting in two dimensions: The critical region
}

\author{
Jeffrey M. Greif and David L. Goodstein \\ California Institute of Technology, 63-37, Pasadena, California 91125 \\ Armando F. Silva-Moreira \\ Instituto de Fisica "Gleb Wataghin," Universidade Estadual de Campinas, \\ 13100 Campinas, Sao Pãolo, Brasil
}

(Received 14 December 1981)

\begin{abstract}
A new analysis is presented of the critical-point behavior of two-dimensional melting in the Kosterlitz-Thouless-Nelson-Halperin-Young theory. The analysis confirms the Kosterlitz-Thouless-Nelson-Halperin-Young critical-point exponent, $\bar{v}=0.36963$. . , but also gives a criterion for its own range of validity amounting to $t<<10^{-3}$, where $t$ is the reduced temperature. Both results are confirmed by direct numerical computation, and it is shown that the corresponding range of correlation lengths is $\xi_{+}>>10^{13}$ lattice spacings. The implications of these results for experimental verification are discussed.
\end{abstract}

\section{INTRODUCTION}

Studies of the statistical mechanics of model two-dimensional (2D) systems have revealed the existence of peculiar low-temperature condensed phases in which conventional long-range order is absent. However, whereas in 3D the absence of long-range order (for instance, in liquids) is characterized by exponentially decaying order-parameter correlations, in 2D systems as diverse as superfluids, ${ }^{1}$ crystals, ${ }^{2}$ and lattices of two-component spins, ${ }^{3}$ what one finds instead are correlations which decay as power laws with temperaturedependent exponents. A significant portion of the recent research in this area has been stimulated by the suggestion of Kosterlitz and Thouless ${ }^{4}$ that transitions out of these unconventional condensed phases are driven by the dissociation of bound pairs of "singularities" which coexist with normal excitations below the transition temperature. For instance, in a 2D superfluid bound vortex-antivortex pairs would coexist with phase fluctuations, in a 2D lattice of two-component spins bound vortex-antivortex pairs would coexist with spin waves, and in a 2D crystal bound dislocation pairs with equal and opposite Burger's vectors would coexist with phonons. On the basis of these ideas, Nelson and Halperin ${ }^{5}$ and Young ${ }^{6}$ have developed a very detailed theory of dislocation-mediated melting in $2 \mathrm{D}$ crystals.

There is considerable current interest in finding experimental systems to which these ideas are applicable. In particular, there are a number of known melting transitions in monolayer adsorbed films which are candidates. In this paper we examine one of the firmest predictions of the theory in the hope of determining whether it is in fact experimentally testable.

At the melting transition, the decay of spatial correlations changes from power law to exponential, with a temperature-dependent correlation length given by

$$
\xi_{+} \sim \exp \left(b /|t|^{\bar{v}}\right)
$$

where $t$ is the reduced temperature, $\left(T-T_{m}\right) / T$, $T_{m}$ being the melting temperature, and $\bar{v}$ is predicted with precision, $\bar{v}=0.369 \ldots$ Experiments consistent with this prediction have recently been reported. ${ }^{7,8}$ We present here an improved analysis of the asymptotic region above the melting transition which yields the same result, but also gives a criterion for the range of validity of this prediction. We are led to expect that it will break down for $t \geq 10^{-3}$. Direct numerical computation confirms this expectation and also shows that a sample larger than $\sim 10^{13}$ lattice spaces would be required to test the prediction. This is a vexing conclusion, since it probably means that the prediction can never be tested experimentally, and that the experiments reporting agreement ${ }^{7,8}$ with it should have been interpreted differently.

In Sec. II of this paper, we outline the relevant parts of the Kosterlitz-Thouless-Nelson-HalperinYoung theory. Our analysis of the asymptotic region is presented in Sec. III and the numerical ver- 
ification in Sec. IV. Conclusions are discussed in Sec. V.

\section{THE KOSTERLITZ-THOULESS-NELSON- HALPERIN-YOUNG THEORY}

The central idea of the Kosterlitz-ThoulessNelson-Halperin-Young theory is that thermally excited dislocation pairs weaken and ultimately destroy the 2D medium's ability to resist shear stress. The renormalization-group procedure is used to take into account the fact that the growth of dislocation pairs promotes the excitation of further pairs. This feedback effect drives the phase transition to completion.

Consider for simplicity a pair of dislocations of oppositely directed Burger's vectors, $\vec{b}_{1}+\vec{b}_{2}=0$, separated by $\vec{r}$. The energy of the pair is given by

$\frac{E_{p}}{k_{B} T}=\frac{b^{2} \bar{K}}{4 \pi} \ln \frac{r}{a}-\frac{\overrightarrow{\mathrm{b}}_{1} \cdot \overrightarrow{\mathrm{r}}_{\mathrm{b}_{2}} \cdot \overrightarrow{\mathrm{r}}}{r^{2}}+2 \frac{b^{2} E_{c}}{k_{B} T}$,

where $a$ is the core diameter and $E_{c}$ the core energy of a dislocation. The quantity $\bar{K}$ depends on the shear modulus $\mu$ and the bulk modulus $B$ according to

$$
\bar{K}=\frac{4 \mu B}{\mu+B} \frac{a_{0}^{2}}{k_{B} T},
$$

where $a_{0}$ is the lattice constant. The probability of finding such a pair in the lattice, $e^{-E_{p} / k_{B} T}$, is proportional to

$$
y^{2}\left(\frac{r}{a}\right)^{-\bar{K} / 4 \pi}
$$

where $y=e^{-E_{c} / k_{B} T}$ acts as a kind of fugacity for the dislocation "gas." A dislocation pair does not greatly weaken the crystal so long as its members are close together and hence tightly bound to each other. At larger separations, however, a pair will more easily polarize and stretch in response to an applied shear stress. The result is that the inverse shear modulus $\mu^{-1}$ increases in proportion to the mean square separation of the pair,

$$
\left\langle\frac{r^{2}}{a^{2}}\right\rangle \propto y^{2} \int\left(\frac{r}{a}\right)^{2}\left(\frac{r}{a}\right)^{-\bar{K} / 4 \pi} d^{2}\left(\frac{\vec{r}}{a}\right)
$$

[ignoring for the moment the angle-dependent term in Eq. (2.1)]. When this integral diverges at $\bar{K}=16 \pi$, the shear modulus has been driven to zero, and the crystal has therefore melted. How- ever, as $\bar{K}$ is reduced toward $16 \pi$ by raising the temperature, the consequent increase in $\left\langle r^{2} / a^{2}\right\rangle$ also tends to reduce $\bar{K}$, making it easier to produce large dislocation pairs. This feedback effect, to be accounted for by means of the renormalization group, ultimately causes $\mu^{-1}$ to diverge discontinuously at the transition.

The classical Hamiltonian for all possible configurations of dislocations is easily written using Eq. (2.1). A configuration of dislocations consists of some set of Burgers vectors located at sites on the 2D lattice. Instead of considering configurations of Burgers vectors on the bare lattice, however, one could consider only those Burgers vectors that appear on a coarser grid. In that case, some pairs of dislocations, with zero net Burgers vector inside of a single element of the coarser grid, would be neglected. Since the neglected pairs would weaken the interaction between those pairs that still appear, the result would be to change the apparent elastic constants, and hence $\bar{K}$ on the coarser grid. In addition, $y$ would change, both because the core diameter associated with a dislocation has changed, and because $E_{c}$ is proportional to $\bar{K}$ :

$$
\frac{E_{c}}{k_{B} T}=\frac{C \bar{K}}{8 \pi} \text {. }
$$

Thus the coarser grid will have a Hamiltonian similar in form to the original lattice, but with new values of $\bar{K}$ and $y$.

The heart of the renormalization group procedure is to find how $\bar{K}$ and $y$ depend on the size of the grid. It is found that when size $a$ is replaced by $a e^{l}, \bar{K}$ and $y$ change according to

$$
\begin{aligned}
\frac{d y(l)}{d l}= & \left(2-\frac{\bar{K}(l)}{8 \pi}\right) y(l) \\
+ & 2 \pi y^{2}(l) e^{\bar{K}(l) / 16 \pi} I_{0}\left[\frac{K(l)}{8 \pi}\right]+O\left(y^{3}(l)\right) \\
\frac{d \bar{K}^{-1}(l)}{d l}= & \frac{3 \pi}{2} y^{2}(l) e^{\bar{K}(l) / 8 \pi} \\
& \times\left[I_{0}\left(\frac{\bar{K}(l)}{8 \pi}\right)-\frac{1}{2} I_{1}\left[\frac{\bar{K}(l)}{8 \pi}\right]\right] \\
& +O\left(y^{3}(l)\right) .
\end{aligned}
$$

Here $I_{0}(x)$ and $I_{1}(x)$ are modified Bessel functions arising from the angle-dependent interaction in Eq. (2.1), and truncation at $O\left(y^{3}\right)$ means we are consid- 
ering only low-density configurations (pairs) of dislocations at each rescaling of the lattice.

Equations (2.4) and (2.5) focus our attention on the coupling constants $\bar{K}$ and $y$. If, as $l$ increases, $y(l)$ is driven to zero, then there are no dislocation pairs at large scales, and the medium is solid. On the other hand, if $y$ diverges, small pairs have made it easy to grow large ones, and the crystal has melted. In addition, the small $y$ approximation made in truncating Eqs. (2.4) and (2.5) becomes invalid, and the theory becomes computationally useless. Depending on the starting point in the $y$ $\bar{K}^{-1}$ plane, Eqs. (2.4) and (2.5) always drive $y$ either to zero or to $\infty$ as $l \rightarrow \infty$.

The starting point in the $y-\bar{K}^{-1}$ plane depends on the temperature and the elastic constants, $\mu_{0}$ and $B_{0}$, of the bare lattice, by way of Eqs. (2.2) and (2.3). The bare elastic constants, in turn, generally depend on the density of the $2 \mathrm{D}$ medium, so a given temperature $T$ and density $N$ (in, say, moles $/ \mathrm{cm}^{2}$ ) correspond to starting coordinates $y_{0}$ and $K_{0}$. Moreover, $y_{0}$ and $K_{0}$ are related through Eq. (2.3),

$$
y_{0}=e^{-C \bar{K}_{0} / 8 \pi} .
$$

Equation (2.6) is known as the line of starting points. For a medium in which the constant $C$, defined by Eq. (2.3), is independent of $N$ and $T$, a single line of starting points represents the entire $N$-T plane. In the $y-\bar{K}^{-1}$ plane, $y \rightarrow 0$ as $l \rightarrow \infty$ for any starting point in a region of small $y$ and $\bar{K}^{-1}$. For $y_{0}$ and $\bar{K}_{0}$ in this region, the medium is solid. This region is bounded by a curve called the separatrix, beyond which $y \rightarrow \infty$ as $l \rightarrow \infty$. The intersection of the line of starting points and the separatrix represents the entire melting curve in the $N-T$ plane, provided $C$ is constant. The $y-\bar{K}^{-1}$ plane, including the separatrix and typical lines of starting points is shown in Fig. 1.

Much attention has been paid to analyzing the behavior of systems with starting points just on the high-temperature side of the separatrix. Here, even though $\bar{K}^{-1}$ and $y$ diverge with increasing $l$, it is still possible to make quantitative predictions by considering the length $\xi_{+}$over which the exponential decay of spatial correlations in the crystal takes place. At any point in this region, there exists a scale sufficiently large that correlations are destroyed in one lattice spacing and $\xi_{+}$is therefore independent of further increase in $l$. It can be shown by a scaling argument that

$$
\xi_{+}=e^{l} \xi_{+}(l) \text {. }
$$

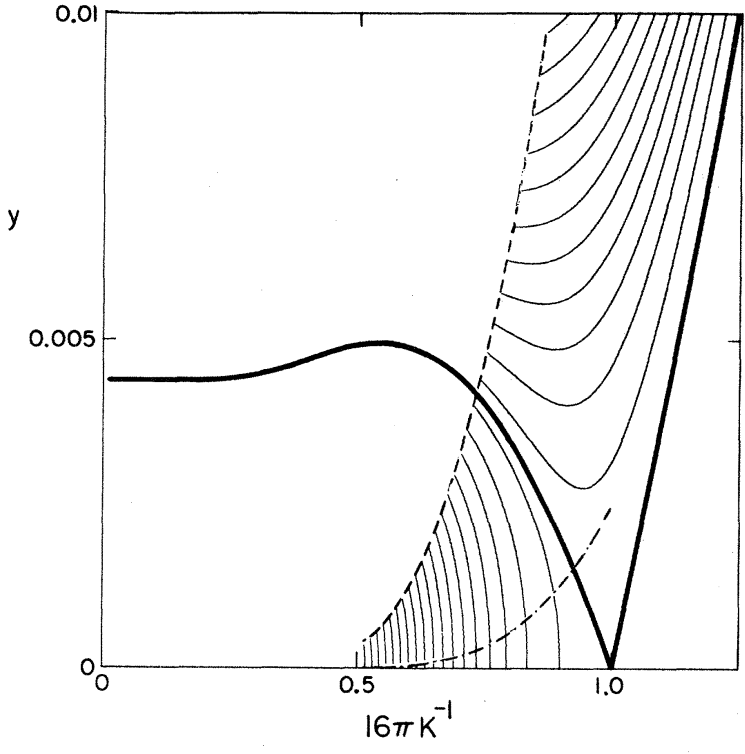

FIG. 1. Phase plane of the renormalization-group equations of Nelson and Halperin for a smooth substrate. Heavy lines represent the separatrices, the dashed line is a line of starting points for $C=2$, and the dot-dashed line shows initial conditions for $C=3$. The remaining curves are renormalization group trajectories. The region below the left separatrix contains all possible combinations of bare elastic constants, temperature, and density for which the system remains solid after accounting for the effect of dislocations.

Thus if $\xi_{+}(l)$ becomes constant at $l>l^{*}$, then

$$
\xi_{+} \sim e^{l^{*}} \text {. }
$$

For starting points sufficiently close to the separatrix, $y\left(l^{*}\right)$ will nevertheless still be small enough so that Eqs. (2.4) and (2.5) are valid. It is then possible to use the theory to find $\xi_{+}$and those quantities that depend on it.

Use of the theory to predict properties of the fluid phase is thus restricted to a region close to the melting point. Under even more restrictive approximations, it has been argued that

$$
l^{*}=\frac{b}{|t|^{\bar{v}}},
$$

where $b$ is a nonuniversal constant, but $\bar{v}$ is known with great precision,

$$
\bar{v}=0.36963 \ldots .
$$

Since an experimental verification of the predictions contained in Eqs. (2.7) - (2.10) would be of considerable significance, it is important to the experimentalist to know over what range of $t$ these results might be expected to be valid. In the next 
section of this paper, we present an improved analysis leading to Eqs. (2.9) and (2.10) which yields a criterion for the range of validity of these equations, namely $t<<10^{-3}$. In the following section we verify this conclusion by direct numerical calculations.

\section{THE CRITICAL EXPONENT AND THE WIDTH OF THE CRITICAL REGION}

In this section we present a new analysis of the behavior in the transition region, which produces the same critical exponent as Nelson and Halperin ${ }^{5}$ and Young ${ }^{6}$ but improves their treatment, and also leads to a criterion for the width of the critical region. In particular, we examine the behavior of the correlation length $\xi_{+}$, as the melting temperature is approached from above.

We determine the correlation length in the critical region by integrating Eqs. (2.4) and (2.5) to some value of the scale $l^{*}$ such that the correlation length is small and slowly varying as a function of l. Then $\xi_{+} \approx e^{l^{*}} \times$ const and $l^{*}$ depends upon the reduced temperature $t$. We will show that $l^{*} \sim|t|^{-\bar{v}}$ where $\bar{v}=0.3693 \ldots$ The scaling law for the correlation length is thus $\xi_{+} \sim e^{b /|t|^{-\bar{v}}}$ where $b$ is a nonuniversal constant.

Near the critical point, $K^{-1}=1 / 16 \pi, y=0$, the separatrix can be regarded as a pair of straight lines with slopes

$$
\begin{aligned}
& m_{ \pm}=\frac{B \pm\left(B^{2}+24 A^{2}\right)^{1 / 2}}{12 \pi A}=\left\{\begin{array}{r}
0.0341 \\
-0.0212
\end{array}\right. \\
& A=e^{2}\left[2 I_{0}(2)-I_{1}(2)\right]=21.937, \\
& B=e I_{0}(2)=6.195,
\end{aligned}
$$

where $I_{n}$ are imaginary Bessel functions. The analysis that follows is restricted to core parameters $C$ large enough so the initial conditions for the integration are in the region near the critical point where the separatrices can be regarded as straight lines. For smaller values of $C$ the same predictions will hold but for an even narrower critical region.

By changing variables to $(x, y)$ with $K^{-1}(l)$ $=(1 / 16 \pi)[1+x(l)]$, Eqs. (2.4) and (2.5) can be written as

$$
\begin{aligned}
& \frac{d x}{d l}=12 \pi^{2} A y^{2}, \\
& \frac{d y}{d l}=2 x y+2 \pi B y^{2} .
\end{aligned}
$$

Defining the deviation from the incoming and outgoing separatrices as

$$
\begin{aligned}
& D(l) \equiv y(l)-m_{-} x(l), \\
& E(l) \equiv y(l)-m_{+} x(l),
\end{aligned}
$$

Eq. (3.1b) can be written as

$$
\frac{d D}{d l}=-2 x D+12 \pi A^{2} m_{+} D^{2}
$$

near the incoming separatrix and

$$
\frac{d E}{d l}=-2 x E+12 \pi A^{2} m_{-} E^{2}
$$

near the outgoing separatrix. Either of the equations is valid anywhere Eq. (3.1b) is, but each is useful only near the appropriate separatrix.

A typical renormalization group ( $R G$ ) trajectory just above the transition is shown in Fig. 1. It hugs first the left and then the right separatrix. The intersection of the line of starting points

$$
y_{0}=e^{-C K_{0} a_{0}^{2} / 8 \pi k_{B} T}
$$

with the trajectory gives the initial conditions $\left(x_{0}, D_{0}\right)$ or $\left(x_{0}, y_{0}\right) . K_{0}$ is the combination of bare elastic constants $4 \mu_{0} B_{0} /\left(\mu_{0}+B_{0}\right)$. The endpoint $\left(x_{1}, E_{1}\right)$ is determined by the criterion for cutting off the $R G$ integral when $\xi_{+}(l)$ is roughly constant. The trajectory passes through a minimum where $d y / d l=0$ in Eq. (3.1b), namely when

$$
y=-\frac{1}{\pi B} x
$$

or equivalently, when

$$
D^{*}=-\left(\frac{1}{\pi B}+m_{-}\right) x^{*}
$$

or

$$
E^{*}=-\left(\frac{1}{\pi B}+m_{+}\right) x^{*} .
$$

In integrating Eqs. (2.4) and (2.5), Eq. (3.2a) is used from the starting point to the minimum, and then Eq. (3.2b) out to the cutoff point. By expanding the line of starting points around the value where it intersects the incoming separatrix, it becomes clear that $D_{0} \propto|t|$.

Equations (3.1) do not depend on $l$ explicitly, so we may consider their quotient

$$
\frac{d x}{d y}=\frac{6 \pi^{2} A y}{x+\pi B y}
$$


which, upon substitution of $y=v x$ can be shown to have the solution

$$
\left(y-v_{+} x\right)^{\alpha}\left(y-v_{-} x\right)^{\beta}=\kappa,
$$

with $v_{ \pm}=m_{ \pm}, \beta=6 \pi^{2} A m_{-}^{2} /\left(1+6 \pi^{2} A m_{-}^{2}\right)$

$=\bar{v}=\overline{0} .36963 .$. , and $\alpha=1-\beta$. Clearly the starting point and endpoint must lie on the trajectory, so

$$
\begin{aligned}
& \kappa=\left(y_{0}-v_{+} x_{0}\right)^{\alpha}\left(y_{0}-v_{-} x_{0}\right)^{\beta}, \\
& \kappa=\left(y_{1}-v_{+} x_{1}\right)^{\alpha}\left(y_{1}-v_{-} x_{1}\right)^{\beta},
\end{aligned}
$$

or in terms of $D$ and $E$

$$
\begin{aligned}
& \kappa=D_{0}^{\beta}\left[D_{0}+\left(v_{-}-v_{+}\right) x_{0}\right]^{\alpha}, \\
& \kappa=E_{1}^{\alpha}\left[E_{1}+\left(v_{-}-v_{+}\right) x_{1}\right]^{\beta} .
\end{aligned}
$$

When $D_{0}$ is sufficiently small and $x_{1}$ sufficiently large that

$$
\begin{aligned}
& D_{0}<<\left|v_{-}-v_{+}\right|\left|x_{0}\right|, \\
& E_{1}<<\left|v_{+}-v_{-}\right| x_{1},
\end{aligned}
$$

a relationship can be established between starting points and endpoints,

$$
E_{1} \approx D_{0}^{\beta / \alpha} \frac{\left(v_{-}-v_{+}\right) x_{0}}{\left[\left(v_{-}-v_{+}\right) x_{1}\right]^{\beta / \alpha}} .
$$

Rewriting the trajectory in terms of $D$ and $E$,

$$
\begin{aligned}
& \kappa=D^{\beta}\left[D+\left(v_{-}-v_{+}\right) x\right]^{\alpha}, \\
& \kappa=E^{\alpha}\left[E+\left(v_{-}-v_{+}\right) x\right]^{\beta},
\end{aligned}
$$

and solving each one for $x$ we find

$$
\begin{aligned}
x & =\frac{\kappa^{1 / \alpha}}{\left(v_{-}-v_{+}\right) D^{\beta / \alpha}}-\frac{D}{\left(v_{-}-v_{+}\right)} \\
& \equiv \frac{\Gamma}{D^{\beta / \alpha}}-\frac{D}{\left(v_{-}-v_{+}\right)} \\
x & =\frac{\kappa^{1 / \beta}}{\left(v_{+}-v_{-}\right) E^{\alpha / \beta}}-\frac{E}{\left(v_{+}-v_{-}\right)} \\
& \equiv \frac{\widetilde{\Gamma}}{E^{\alpha / \beta}}-\frac{E}{\left(v_{+}-v_{-}\right)}
\end{aligned}
$$

where the constants $\Gamma$ and $\widetilde{\Gamma}$ contain all the dependence on initial or final conditions. When the approximations of Eqs. (3.5) are valid,

$$
\begin{aligned}
& \Gamma \approx D_{0}^{\beta / \alpha} x_{0}, \\
& \widetilde{\Gamma} \approx E_{1}^{\alpha / \beta} x_{1} .
\end{aligned}
$$

Inserting Eqs. (3.6) into Eqs. (3.2) we find

$$
\frac{d D}{d l}=-2 \Gamma D^{1-\beta / \alpha}+G D^{2},
$$

$$
\frac{d E}{d l}=-2 \widetilde{\Gamma} E^{1-\alpha / \beta}+H E^{2},
$$

where

$$
\begin{aligned}
& G \equiv 12 \pi^{2} A m_{+}+\frac{2}{\left(v_{-}-v_{+}\right)}, \\
& H \equiv 12 \pi^{2} A m_{-}+\frac{2}{\left(v_{+}-v_{-}\right)} .
\end{aligned}
$$

Now the integral of the renormalization group trajectory is the sum of two quadratures with no closed form solution, i.e.,

$$
l^{*}=l_{1}^{*}+l_{2}^{*},
$$

where

$$
\begin{aligned}
& l_{1}^{*}=\int_{D_{0}}^{D^{*}} \frac{d D}{-2 \Gamma D^{1-\beta / \alpha}+G D^{2}}, \\
& -l_{2}^{*}=\int_{E_{1}}^{E^{*}} \frac{d E}{-2 \widetilde{\Gamma} E^{1-\alpha / \beta}+H E^{2}} .
\end{aligned}
$$

How does $l^{*}$ depend on $|t|$ ?

It is tempting to discard the second term in the denominator of each integral on the grounds that for small $D$ and $E$, the first term dominates. For small $D_{0}$ and $E_{1}, D$ and $E$ are small near the endpoints, but, as we now show, become larger near the minimum $\left(D^{*}\right.$ and $\left.E^{*}\right)$, where, in fact, both terms in the denominator of each integral are of similar size.

Inserting Eqs. (3.6) into Eqs. (3.4) we find solutions

$$
\begin{aligned}
& D^{*}=R \Gamma^{\alpha} \sim D_{0}^{\beta}, \\
& E^{*}=S \widetilde{\Gamma}^{\beta} \sim E_{1}^{\alpha},
\end{aligned}
$$

with $R$ and $S$ constants independent of $D_{0}$ and $E_{1}$. Comparing Eq. (3.8a) and Eq. (3.8b) we find that

$$
D_{0}^{\beta} \sim E_{1}^{\alpha} \text {. }
$$

Rearranging terms in Eqs. (3.7) we find the denominators at the minimum,

$$
\Gamma^{2} \alpha\left(-2 R^{1-\beta / \alpha}+G R^{2}\right)
$$

and

$$
\widetilde{\Gamma}^{2} \beta\left(-2 S^{1-\alpha / \beta}+H S^{2}\right),
$$

so the dependence upon starting point factors out. The constants in Eq. (3.9), for example, are nearly the same

$$
\frac{R^{1-\beta / \alpha}}{R^{2}}=50.567, G=76.08,
$$

so the integrals for $l^{*}$ must be evaluated without 
truncating the denominator.

This is done by rescaling the equations to remove the dependence on $D_{0}$ from the integrand. Let $P \equiv D / D_{0}^{s}$ and determine $s$ such that all the dependence upon $D_{0}$ factors out of the integral in Eq. (3.7a). Not surprisingly, $s=\beta$. Similarly, the substitution $Q \equiv E / E^{\alpha}$ produces the following set of integrals

$$
\begin{aligned}
& l_{1}^{*}=D_{0}^{-\beta} \int_{P_{0}}^{P^{*}} \frac{d P}{-2 x_{0} P^{1-\beta / \alpha}+G P^{2}}, \\
& -l_{2}^{*}=E_{1}^{-\alpha} \int_{Q_{0}}^{Q^{*}} \frac{d Q}{-2 x_{1} Q^{1-\alpha / \beta}+H Q^{2}},
\end{aligned}
$$

where Eqs. (3.5) have been used to simplify $\Gamma$ and $\widetilde{\Gamma}$. Now differentiate the integrals with respect to $D_{0}$ and $E_{1}$, respectively, to find

$$
\begin{aligned}
& \frac{d l_{1}^{*}}{d D_{0}}=\frac{-\beta l_{1}^{*}}{D_{0}}-D_{0}^{-\beta} \frac{\alpha D_{0}^{-\beta}}{-2 x_{0} P_{0}^{1-\beta / \alpha}+G P_{0}^{2}}, \\
& \frac{-d l_{1}^{*}}{d E_{1}}=\frac{-\alpha l_{2}^{*}}{E_{1}}-E_{1}^{-\alpha} \frac{\beta E_{1}^{-\alpha}}{-2 x_{1} Q_{1}^{1-\alpha / \beta}+H Q_{1}^{2}},
\end{aligned}
$$

since

$$
P_{0}=D_{0} / D_{0}^{\beta}=D_{0}^{\alpha}
$$

and similarly,

$$
Q_{1}=E_{1}^{\beta} \text {. }
$$

The second term in each of the differential equations (3.11) above arises from differentiating the lower limit of the integrals in Eqs. (3.10). The upper limit makes no contribution, because by choice of $P$ and $Q$ and Eqs. (3.8), $P^{*}$ and $Q^{*}$ are independent of $D_{0}$ and $E_{1}$, respectively.

Inserting Eqs. (3.12) into Eqs. (3.11), the differential equations can be solved by using integrating factors. One finds

$$
\begin{aligned}
& l_{1}^{*}=D_{0}^{-\beta} \int D_{0}^{D_{0}} \frac{\alpha d \Delta}{\Delta^{\alpha}\left(-2 x_{0}+G \Delta\right)}, \\
& -l_{2}^{*}=E_{1}^{-\alpha} \int^{E_{1}} \frac{\beta d \epsilon}{\epsilon^{\beta}\left(-2 x_{1}+H \epsilon\right)} .
\end{aligned}
$$

The second term in the denominators of Eqs. (3.13) can now be discarded, for suitably small values of $D_{0}$ and $E_{1}$, and the integrals yield

$$
l_{1}^{*} \approx \frac{\alpha}{-2 x_{0} \beta}+M D_{0}^{-\beta}
$$

$$
l_{2}^{*} \approx \frac{\beta}{2 x_{1} \alpha}+N E_{1}^{-\alpha}
$$

where $M$ and $N$ are constants. Since $E_{1} \sim D_{0}^{\beta / \alpha}$, we have

$$
l^{*}=l_{1}^{*}+l_{2}^{*} \sim D_{0}^{-\beta} \sim|t|^{-\beta}
$$

which agrees with the result of Nelson and Halperin. $^{5}$

The present calculation improves that of Nelson and Halperin because their analysis of the behavior of $l^{*}$ worked by means of an incorrect approximation. They integrated from the initial point toward the minimum, and stopped when $D$ became significant (before the minimum), then arbitrarily jumped across the minimum and added a piece gained from integrating along the outgoing separatrix. We have found in the course of the numerical calculation presented in Sec. IV that nearly all the contribution to $l^{*}$ comes near the minimum. Fortunately that part also $\sim|t|^{-\beta}$ so that Nelson and Halperin got the correct dependence (they would have obtained the wrong constants of proportionality had they tried to determine them).

The only approximations in the treatment above are those of Eqs. (3.5) and the truncation of the denominator of Eqs. (3.13). (These latter could be removed by performing a Taylor series expansion to any desired accuracy, but we are only concerned with the leading order behavior.) The approximations produce bounds on the validity of the scaling law, and hence give an indication of the width of the critical region.

From Eqs. (3.5) we find

$$
\begin{aligned}
& D_{0}<<\left|0.0564 x_{0}\right|, \\
& E_{1}<<\left|0.0564 x_{1}\right| \text { or } D_{0}<<\left|0.0564 x_{1}\right|^{(1-\beta) / \beta}
\end{aligned}
$$

and from the integrals,

$$
G D_{0}<<2 x_{0} \text { or } D_{0}<<0.0341 x_{0},
$$

and

$$
H E_{1}<<2 x_{1} \text { or } D_{0}<<\left(0.0223 x_{1}\right)^{(1-\beta) / \beta} \text {. }
$$

Clearly, the controlling approximations are Eqs. (3.14a) and (3.14b). Since $(1-\beta) / \beta=1.71$, we can rewrite Eq. (3.14b) as

$$
D_{0}<<0.0015 x_{1}^{1.71} \text {. }
$$

Choosing $x_{1}=0.1$ (supposedly far enough from the transition that $\xi_{+}$is roughly constant) and $x_{0}=-0.1$, the controlling approximation is Eq. $(3.14 \mathrm{c})$, 


$$
D_{0}<<2.9 \times 10^{-5} \text {. }
$$

It remains only to find the proportionality between $D_{0}$ and $|t|$, from the equation of the line of starting points. Expanding Eq. (3.3) around the intersection with the separatrix we obtain

$$
\begin{aligned}
& y_{0} \approx y_{s}\left(1+\frac{C K_{0} a_{0}^{2}}{8 \pi k_{B} T_{s}} t\right), \\
& x_{0} \approx x_{s}+\frac{K_{0} a_{0}^{2}}{16 \pi k_{B} T_{s}} t,
\end{aligned}
$$

where the subscript $s$ refers to values at the intersection of the line of starting points with the separatrix,

$$
y_{s}=m_{-} x_{s} .
$$

Thus,

$$
D_{0}=y_{0}-m_{-} x_{0}=Q_{s} t\left(y_{s}-\frac{m_{-}}{2 C}\right),
$$

where $Q_{s}=C K_{0} a_{0}^{2} / 8 \pi k_{B} T_{s}$. The factor multiplying $t$ in Eq. (3.17) is only weakly dependent on $C$ in the range where our approximation of a linear separatrix is valid, since in this range, $y_{s}$ is small compared to $m_{-} / 2 C$, and $Q_{s} \approx 2 C$. Taking $C \approx 3$ so that $Q_{s} \approx 6$ and neglecting $y_{s}$, we find $D_{0} \approx 0.03 t$, which when combined with Eq. (3.15) yields

$$
t<<10^{-3} \text {. }
$$

This restriction applies to $C \gtrsim 2$, where the separatrix is linear. However, a given starting point in this region can be reached along a renormalization group trajectory from a starting point at lower $C$ only by choosing an even smaller reduced temperature. Thus the restriction is completely general.

\section{NUMERICAL SOLUTION OF THE RG EQUATION}

The conclusions of Sec. III are that Eqs. (2.9) and (2.10) should be obeyed for $t<<10^{-3}$. In this section we describe numerical calculations which confirm those conclusions and which also show that (2.9) and (2.10) are not obeyed if $t$ is larger than $10^{-3}$.

The basic idea of the calculation is to start from a point in the $y-\bar{K}^{-1}$ plane corresponding to a known value of $t$, integrate Eqs. (2.4) and (2.5) until an appropriate value of $l^{*}$ is reached, then compare the resulting $l^{*}(t)$ to the prediction, Eqs. (2.9) and (2.10). The procedure is straightforward, but some discussion is needed concerning both the starting points and end points of the integrations.

For a given choice of $C$, the line of starting points, Eq. (2.6) intersects the separatrix at some point $y_{s}, \bar{K}_{s}^{-1}$. If the integration begins from a nearby point on the line of starting points, $y_{0}, \bar{K}^{-1}$, the calculation will correspond to a reduced temperature.

$$
t=\frac{T-T_{m}}{T_{m}}=\frac{\bar{K}_{0}^{-1}-\bar{K}_{s}^{-1}}{\bar{K}_{s}^{-1}} .
$$

Since we are interested in small values of $t$, the intersection, and hence the separatrix itself must be known with high numerical precision.

To find the separatrix, Eq. (2.4) is divided by Eq. (2.5) to produce an expression for $d y / d \bar{K}^{-1}$. This equation is then integrated starting from the critical point $\left(y=0, \bar{K}^{-1}=1 / 16 \pi\right)$ along a curve whose initial slope is given by $m_{-}$(as discussed in Sec. III). The result traces out the separatrix, shown in Fig. 1. The result is checked by picking starting points $y, \bar{K}^{-1}$ on the presumed separatrix and integrating in the reverse direction, to see that the critical point is reached. A starting point slightly above the separatrix results instead in $y$ diverging.

As seen in Fig. 1, the resulting separatrix has a maximum at $16 \pi \bar{K}^{-1}=0.55$ of $y=0.497 \times 10^{-2}$, and its value at $16 \pi \bar{K}^{-1}=0$ is $y=0.437 \times 10^{-2}$. Knowledge of the separatrix is useful for extracting a number of predictions from the theory. For example, if a 2D solid at a certain density is known to have elastic constants $\mu_{0}$ and $B_{0}$ at $T<<T_{m}$, the theory predicts

$$
T_{m}=\frac{16 \pi}{\bar{K}_{s}} \frac{4 \mu_{0} B_{0}}{\mu_{0}+B_{0}} \frac{a_{0}^{2}}{K_{B}},
$$

where $\bar{K}_{s}$ depends on $C$ as described above.

We have computed $l^{*}(t)$ for the lines of starting points given by $C=2$ and 3 . These are arbitrary choices, since there does not seem to be a dependable theory of dislocation core energies. For these values, the intersection coordinates are given in Table I.

TABLE I. Intersection coordinates for $C=2$ and 3.

\begin{tabular}{lcc}
\hline \hline$C$ & $16 \pi / \bar{K}_{s}$ & $10^{2} y_{s}$ \\
\hline 2 & $0.730 \ldots$ & $0.418 \ldots$ \\
3 & $0.924 \ldots$ & $0.151 \ldots$ \\
\hline
\end{tabular}


TABLE II. Values of $l^{*}$ for the various cutoff criteria, for $C=2$.

\begin{tabular}{ccrc}
\hline \hline & & Criterion & \\
$t$ & $16 \pi \bar{K}^{-1}=1.1$ & $y=0.1$ & $y$ diverges \\
\hline $10^{-6}$ & 469.0 & 473.5 & 474.0 \\
$10^{-5}$ & 195.0 & 199.0 & 200.0 \\
$10^{-4}$ & 78.0 & 82.4 & 83.0 \\
$10^{-3}$ & 29.0 & 33.0 & 34.0 \\
\hline \hline
\end{tabular}

Once the intersection has been established, the starting point for integrating the $R G$ equations fixes the value of $t$. The end point is $l=l^{*}$, where $l^{*}$ is the object of the calculation. It should be chosen, as discussed in Sec. II, such that $\xi_{+}(l)$ becomes small and independent of $l$ for $l>l^{*}$. However, since $\xi_{+}(l)$ is not known, a different criterion must be used for terminating the integration.

It has variously been suggested ${ }^{9}$ that the integration be stopped at an arbitrary value of $\bar{K}^{-1}$, say, $16 \pi \bar{K}^{-1}=1.1$, or at a value of $y$, say, $y=0.1$. We have found it convenient for computational purposes to stop the integration routine when $y$ starts to diverge rapidly. It is very much to the point that any of these procedures would give essentially the same result for $\log \left(l^{*}\right)$. That this is so is shown in Table II.

It was found in doing the calculation that the results were undependable for $l \geq 500$, due to an un-

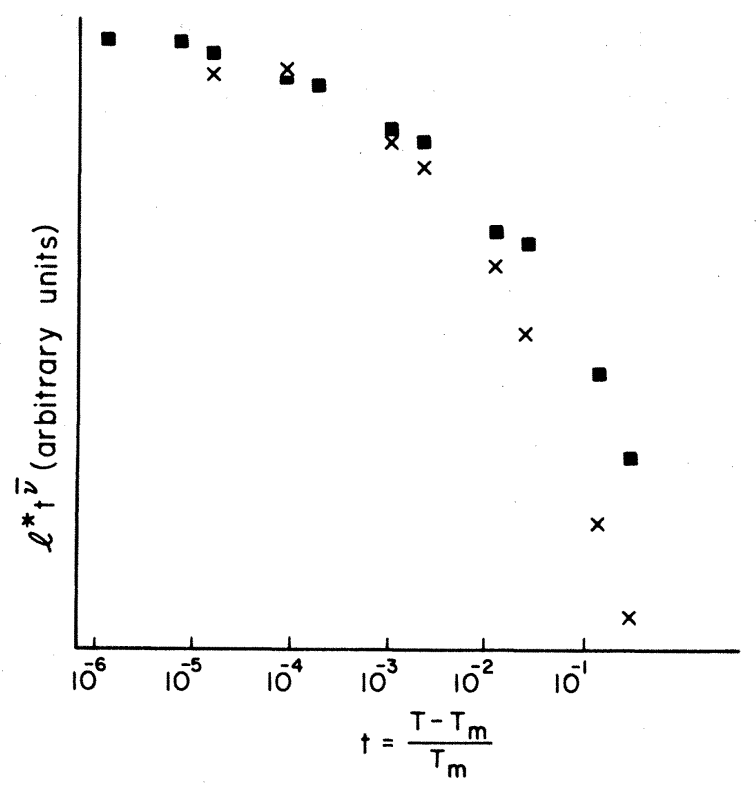

FIG. 2. Normalized $l^{*} t^{\bar{v}}$ vs $\log t$ for $C=2(\times)$ and $C=3(\mathbb{a})$ from numerical integration of $R G$ equations. known difficulty in the algorithm. Restricting ourselves to $l \leq 500$, it was possible to test the predictions for $t \gtrsim 10^{-5}$ when $C=3$ and $t \geq 10^{-6}$ when $C=2$. The results are displayed in a plot of $t^{\bar{v}} l^{*} / l^{*}\left(t=10^{-5}\right)$ vs $\log _{10} t$ in Fig. 2 .

As seen in Fig. 2, the calculations confirm the analysis of Sec. III. The curves of $t^{\bar{\nu}} l^{*}$ vs $\log t$ become horizontal only when $t \ll 10^{-3}$. For $t \leq 10^{-3}$, they depart from that behavior, meaning that the asymptotic prediction for the behavior of $\xi_{+}$is no longer being obeyed.

\section{CONCLUSIONS}

Experimental verification of the predicted critical-point behavior of 2D melting would require a very precise determination of the melting temperature $T_{m}$ in order that the critical region, $t<10^{-3}$ might be examined. To our knowledge, there is no instance in which $T_{m}$ has been determined with the required precision for a melting transition (nor indeed for any experimental instance of what is presumed to be a Kosterlitz-Thouless-type transition). In fact, the theory itself presents formidable barriers to determining the transition temperature precisely.

$T_{m}$ is the lowest temperature at which $y$ and $\bar{K}^{-1}$ diverge as $l \rightarrow \infty$. Thus $T_{m}$ cannot be determined exactly in any experiment sensitive to the

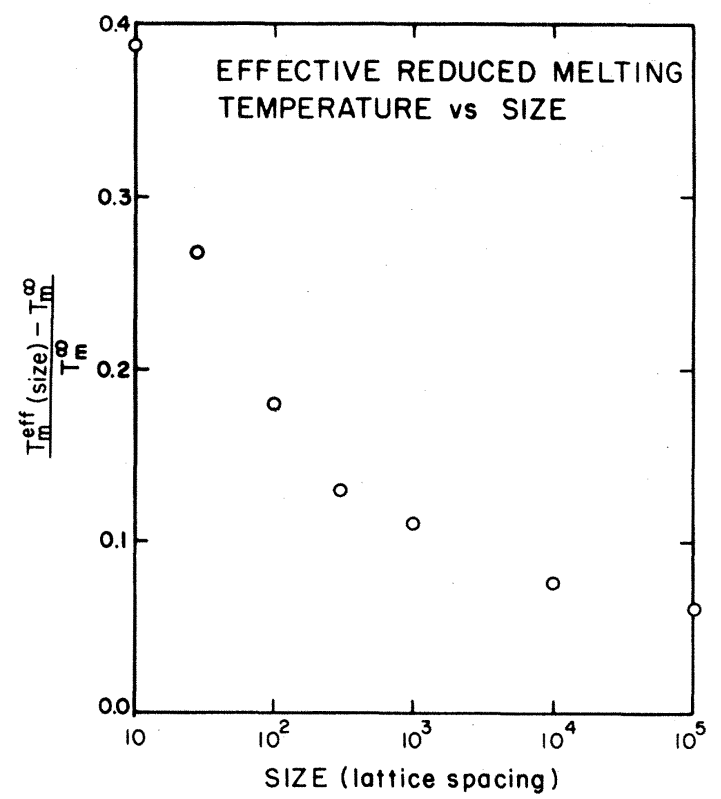

FIG. 3. Apparent reduced melting temperature (relative to that for an infinite medium) for a patch of given size (in the infinite medium). 
behavior of the system on finite scales. Correspondingly, dynamical experiments do not determine the exact transition temperature if they probe finite frequencies.

A quantitative estimate of the difficulty may be obtained using the data in Table II. To observe critical-point behavior we must have $t \leqslant 10^{-3}$ which means a correlation length larger than $e^{30} \approx 10^{13}$ lattice spacings. On smaller length scales the medium remains solid until a higher temperature is reached. Experiments ${ }^{7,8}$ are more typically done on samples whose linear dimensions are of order $10^{2}-10^{7}$ lattice spacings. Thus in practical cases, the effective melting temperature occurs well outside the range where the exponent $\bar{v}$ might play any role.

Even if the sample is very large, the nature of the experiment must be taken into account. For example, a neutron scattering experiment, in order to detect critical-point behavior, would need to have momentum transfer wave vectors shorter than $\sim 10^{-13}$ (lattice spacings) $^{-1}$. An analogous requirement on frequencies means that experiments must be done on very long time scales.
Since many experiments are done on what are in practice very small samples (i.e., Grafoil), we have plotted in Fig. 3 the apparent melting temperature as a function of sample size using parameters (elastic constants) applicable to ${ }^{4} \mathrm{He}$ with an assumed core energy parameter $C=2.8$. A realistic treatment of the problem would have to take into account conditions at the sample boundary and other complications; Fig. 3 indicates only when the infinite sample correlation length becomes smaller than the sample size, but it does give some feeling for magnitudes in the problem.

To be sure, the theory does yield other predictions which may be testable in certain circumstances. However, the critical-point behavior under consideration here would appear to be rather less important than is suggested by the very considerable theoretical attention it has received.

This work was supported by Brazilian National Research Council (CNPq), DOE Grant No. DEAM03-76SF00767, and Cooperative NSF Grant Brazil U. S. No. INT8023690.
1P. C. Hohenberg, Phys. Rev. 158, 383 (1967).

${ }^{2}$ N. Mermin, Phys. Rev. 176, 250 (1968).

${ }^{3}$ N. D. Mermin and H. Wagner, Phys. Rev. Lett. 17, 1133 (1966).

4J. M. Kosterlitz and D. J. Thouless, J. Phys. C $\underline{6}, 1181$ (1973).

${ }^{5}$ D. R. Nelson and B. I. Halperin, Phys. Rev. B $\underline{19}$, 2457 (1979).
${ }^{6}$ A. P. Young, Phys. Rev. B $\underline{19}, 1855$ (1979).

${ }^{7}$ P. A. Heiney, R. J. Birgeneau, G. S. Brown, P. M. Horn, D. E. Moncton, and P. W. Stephens (unpublished).

${ }^{8}$ J. P. McTague, J. Als-Nielsen, J. Bohr, and M. Nielsen (unpublished).

${ }^{9}$ D. R. Nelson, Phys. Rev. B $\underline{18}, 2318$ (1978). 